\title{
Production of neutrals and their effects on the ion charge states in cathodic vacuum arc plasmas
}

André Anders ${ }^{a}$, Efim M. Oks ${ }^{b}$, and Georgy Yu. Yushkov

a) Lawrence Berkeley National Laboratory, University of California, 1 Cyclotron Road, Berkeley, California 94720

b) High Current Electronics Institute, Russian Academy of Sciences, 4 Academichesky Ave., Tomsk 634055, Russia

\begin{abstract}
Cathodic arc plasmas are considered fully ionized and they contain multiply charged ions, yet, gaseous and metal neutrals can be present. It is shown that they can cause a significant reduction of the ion charge states as measured far from the cathode spots. Several cathode materials were used to study the evolution the mean ion charge state as a function of time after arc ignition. The type of cathode material, arc current amplitude, intentionally increased background gas, additional surfaces placed near the plasma flow, and other factors influence the degree of charge state reduction because all of these factors influence the density of neutrals. In all cases, it was found that the mean ion charge state follows an exponential decay of first order, $\bar{Q}(t)=A \exp (-t / \tau)+\bar{Q}_{s s}$, where $A$ is a parameter describing the importance of the decay, $\tau$ is the characteristic decay time, and $\bar{Q}_{s s}$ is a steady-state value approached for continuous arc operation. The extrapolated values $\bar{Q}(t \rightarrow 0)$ indicate surprisingly high mean charge states as produced at cathode spots and not "skewed" by charge exchange collisions with neutrals.
\end{abstract}




\section{INTRODUCTION}

For many years it has been known that cathodic arc plasmas go through an initial transition phase until they reach noisy steady-state conditions. The fluctuations or "noise” of plasma parameters can be traced back to the non-stationary cathode processes that produce plasma. While it is firmly established that cathodic arc spot processes are fast, often down to the resolution limit of nanoseconds, ${ }^{1-5}$ the duration of the initial transition phase is orders of magnitude longer, namely 100 s of microseconds ${ }^{6}$ or even milliseconds. ${ }^{7}$ Several ideas have been brought forward to explain the phenomenon. For example, it was speculated that the surface conditions may have slowly changed due to the temperature evolution of the cathode surface, and the chemical state of the cathode surface may have changed as the result of residual gas bombardment on the one hand and erosion on the other. ${ }^{6}$

In previous contributions ${ }^{8,9}$ it was pointed out that the metal plasma produced at cathode spot interacts with the neutral gas or vapor in the pre-cathode region. The neutral "background” consists of (a) gas molecules from the residual or intentional background gas, (b) gas desorbed from walls and other components due to the interaction with plasma, and (c) metal atoms generated by the arc operation itself. There are several sources of metal neutrals, including evaporating macroparticles, ${ }^{10}$ hot surface areas (craters) from previously active cathode spots, and the incomplete energetic plasma condensation process when the sticking coefficient is less than unity and the self-sputter yield is greater than zero. ${ }^{11}$ Hot neutrals are produced by the latter two mechanisms, whereas the other produce thermal neutrals with temperature approximately equal to the temperature of the evaporating surface. The presence of neutrals in the flowing plasma leads to charge exchange collisions which 
have an effect on all plasma properties, and especially on the ion charge state and velocity distribution functions.

Our recent publication on $\mathrm{Bi}$ and $\mathrm{Pb}$ plasmas gave strong evidence that neutrals evaporating from the cathode are responsible for the shifts in the charge state distribution. ${ }^{9}$ The implication was that the ion charge states, as produced at cathode spots, are actually higher than generally reported. Here we consider a greater number of different materials and try to address the questions: Are non-sticking of metal and evaporation significant sources of neutrals for most or all cathode materials? Are both gas molecules and metal atoms relevant for the evolution of the transient stage towards steady-state? If yes, what is their relative relevance? Can we derive the original charge states as produced by cathode spots, i.e., before they are "skewed" by the interaction with neutrals?

\section{EXPERIMENTS}

\section{A. General Setup}

The experiments were done with the vacuum arc metal ion source "Mevva-V" at Berkeley Lab; this source has been previously described ${ }^{12}$ and therefore it is sufficient to only briefly summarize its features. It consists of a plasma source, a three grid ion extraction system, and a time-of-flight (TOF) mass/charge spectrometer. ${ }^{13}$ Via a "gun barrel" mechanism one can select one of the up to 18 mounted cathodes for cathodic arc operation. Each cathode was a metal rod of $1 / 4$ inch $(6.25 \mathrm{~mm})$ diameter and about 1 inch $(25 \mathrm{~mm})$ length, inserted in an alumina tube. Cathode spots could only burn on the rod's front surface, with the metal plasma expanding through an annular anode of $1 / 2$ inch $(12.5 \mathrm{~mm})$ inner diameter into a plasma expansion chamber that had about $10 \mathrm{~cm}$ diameter and $10 \mathrm{~cm}$ length. 
The plasma streams from the cathode spots towards the extraction grid system. Ions were extracted from the plasma by a three-grid multi-aperture system of the accelerationdeceleration type. ${ }^{14}$ The suppressor grid voltage was $-2 \mathrm{kV}$ and the extraction voltage was 40 $\mathrm{kV}$. The TOF instrument has a drift length of $1.03 \mathrm{~m}$ and the detector was a magnetically suppressed Faraday cup.

Different metals ( $\mathrm{Al}, \mathrm{Mg}, \mathrm{Cu}, \mathrm{Pb}, \mathrm{Bi}, \mathrm{Mo}$ ) were used as cathode in the experiments. The charge state distributions were measured at selected delay times after arc triggering using the time-of-flight (TOF) spectrometer. Each arc discharge of typically 300 A had a duration of $300 \mu$ s, determined by the pulse-forming network, at a repetition rate of up to 10 pulses per second (pps). This high repetition rate ensured that, under base pressure conditions of about $10^{-4} \mathrm{~Pa}$, a gas layer cannot form on the cathode surface between arc pulses. For some experiments, the arc pulse repetition rate and arc current were changed to study the effects of these changes.

\section{B. Experiments with background gas}

The effects of gas and metal vapor were studied by (i) introducing nitrogen gas in the cathode region and (ii) by intentionally introducing surfaces with which the expanding metal plasma can interact (see point C below). Upon introduction of gas near the cathode, enhanced pressure was essentially limited to the region between cathode and extraction grid system because the extraction grid system acted as an array of flow-limiting orifices. The cryogenic pump, with a pumping speed of $1500 \mathrm{l} / \mathrm{s}$ for nitrogen, was mounted to the main chamber that housed the TOF instrument; the extraction grid system reduced the pumping speed, and thereby the TOF instrument was differentially pumped. 


\section{Modified Geometries}

For the study of the metal neutrals produced by the interaction of plasma with surfaces, three cases were considered: (1) the original ion source geometry, where the plasma expansion occurred in the previously mentioned expansion chamber, (2) a set of annular baffles made from $0.5 \mathrm{~mm}$ thick aluminum sheet metal, with an inner diameter of $40 \mathrm{~mm}$ and spaced $5 \mathrm{~mm}$ apart from each other, inserted in the expansion zone between anode and extraction grid; and (3) a aluminum tube of the same length and inner diameter as the baffle system. The idea of using these different surfaces in the expansion zone was to affect the production of neutrals by non-sticking of ions and self-sputtering, in the case these processes matter. When using the baffles, the desorbed gas would fill the whole expansion zone, whereas metal neutrals produced by non-sticking would most likely move to the opposing baffle surface and very likely condense there, thus being removed form the plasma system.

Finally, we also changed the distance between cathode (location of ion production) and extraction grid (location of ion analysis). For this experiment, the standard 18-cathode holder was removed and a movable, single cathode plasma source installed (“minigun”15). The cathode size was the same as with the 18-cathode source.

\section{RESULTS}

In the first round of experiments we used $\mathrm{Al}, \mathrm{Mg}, \mathrm{Cu}, \mathrm{Pb}$, and $\mathrm{Bi}$ as cathodes in the conventional ion source configuration without intentional gas introduction. As expected, one obtains curves of falling mean ion charge states, $\bar{Q}(t)$, as shown in Fig. 1. The decay of the mean charge states can be fitted with first-order exponential functions of the form 


$$
\bar{Q}(t)=A \exp (-t / \tau)+\bar{Q}_{s s}
$$

where $A$ is a factor that describes the relevance of the decay, $\tau$ is the characteristic decay time, and $\bar{Q}_{s s}$ is the steady-state average charge state number which is approached for $t \gg \tau$. The first order exponential decay offers a surprisingly good fit, as one can see from the correlation coefficient in Table I. The strongest decay is seen with Bi, which was attributed to the very low thermal conductivity of this material, leading to higher cathode surface temperatures and, given the high vapor pressure of this material, a strong generation of neutral metal vapor. ${ }^{9}$ The vapor in turn leads to charge exchange collisions, reducing especially the higher charge states, as will be discussed later.

Figure 2 shows a compilation of results of charge state measurements for copper ions under various geometrical and pressure conditions. The elevated pressures were determined from measurements downstream of the grounded extractor electrode and not at the high potential $(40 \mathrm{kV})$ inside the plasma generator. The pressure values mentioned in figure 2 refer to the pressure in the plasma expansion zone and take differential pumping into account. From the figure we can see that (i) the baffles and tube inserted in the expansion zone reduce the mean ion charge state, (ii) the tube has an even greater effect than the baffles, (iii) background gas reduces the mean ion charges, and the reduction is stronger with increasing pressure, and (iv) at sufficiently high gas pressure, the mean ion charge is reduced from the very beginning of the pulse and not further reduced as the arc pulse develops.

Previous measurements have shown that at low arc pulse repetition rates, the plasma contains large amounts of non-metal ion species such as hydrogen and oxygen, and that the ion charge states are reduced compared to high-repetition rate operation. ${ }^{16}$ Now, checking the reduction behavior in terms of its exponential decay and the correlation with gaseous and 
metallic neutrals, we used aluminum to reproduce the effect (Fig. 3). One can clearly discern the lower metal charge states and qualitatively different behavior when a long time elapses between arc pulses. For a quantitative evaluation one can again use an exponential decay of first order; the fit data are given in Table II.

The same experiment with a molybdenum cathode also shows the influence of the pulse repetition rate (Fig. 4 and Table III) but it is much less pronounced compared to aluminum. However, there is a clear influence of the arc current, as shown in Figures 5(a) and (b) for arc repetition rates of 1 and 10 pulses per second, respectively; the characteristic data are compiled in Table IV.

A critical test of the hypothesis of charge exchange collisions is to check the dependence of the charge state reduction on the path length ions have to travel before they are extracted and analyzed. For this, the cathode assembly was mounted on a movable feedthrough. The result is presented in Fig. 6 and Table V, where one can clearly see that the greater the distance the lower the mean ion charge state.

\section{DISCUSSION}

The compilation of data allows us to return to the questions posed at the end of the Introduction. First, one can state that the reduction of ion charge states after arc ignition is a universal phenomenon (Fig. 1), however, the speed and significance of this reduction depend on the cathode material and are affected by numerous parameters such as the presence of background gas, the time the cathode was exposed to the background gas before arc ignition, the arc current, etc. The placement of additional surfaces (baffles or tube) reduced the ion charge states, and the effect was more pronounced for the tube (Fig. 2). In all cases, plasma 
is interacting with surfaces, desorbing gas and, additionally, creating metal neutrals because the metal ion sticking probability is less than unity and the self-sputtering yield is greater than zero, especially for higher ion energies and off-normal incident angles. ${ }^{8,11,17}$ As expected, the effect of the tube is somewhat greater because all of the neutrals produced are directed back into the volume where the plasma streams toward the extraction grid (ion analysis). In the case of baffles, some of the neutrals may bounce off away from the plasma flow and may gettered at the surface of the chamber and therefore do not interact with the ions that are analyzed.

With the intentional presence of background gas, the charge states are reduced compared to the vacuum case (Fig. 2). The reduction is directly related to the gas pressure and not delayed but affected immediately, supporting the notion that — in the vacuum case - the evolution of neutrals is responsible for the evolution of the charge state distribution.

The flux of ions of charge state $Q$ emitted from active cathode spots, $\Gamma_{Q}$, will be reduced by ${ }^{18}$

$$
d \Gamma_{Q}=-\sigma_{Q a} \Gamma_{Q} n_{a} d z
$$

where $z$ is the coordinate normal to the cathode surface, $n_{a}$ is the density of the vapor atoms, $\sigma_{Q a}$ is the cross section for interaction of the ion with neutral atoms or molecules. If $\sigma_{Q a}$ is independent of position one obtains

$$
\Gamma_{Q}(z)=\Gamma_{Q}(0) \exp \left(-z / \lambda_{Q}\right)
$$

with the mean free path

$$
\lambda_{\mathrm{Qa}}=\left(n_{a} \sigma_{\mathrm{Qa}}\right)^{-1} .
$$

The ion-neutral interaction is dominated by charge exchange collisions of the type 


$$
M^{Q+}+M \rightarrow M^{(Q-1)+}+M^{+}+\Delta E
$$

where $\Delta E$ is the energy defect. For $Q=1$ the collision is symmetric and $\Delta E=0$ (resonant charge exchange). The special case $Q=1$ does not reduce the mean ion charge state,

$$
\bar{Q}=\sum_{Q^{\prime}=1}^{Q_{\max }^{i}} Q^{\prime} n_{Q^{\prime}} / \sum_{Q^{\prime}=1}^{Q_{\max }^{i}} n_{Q^{\prime}},
$$

however, all collisions (5) involving multiply charged ions do. One should note that although charge is conserved in all reactions, the mean ion charge state is reduced because the summation in (6) does not include neutrals. All reactions of the type (5) for $Q>1$ produce singly ionized ions from neutrals, thereby increasing the number of singly charged ions while reducing the number of multiply charged, i.e., the mean ion charge state is reduced.

Charge transfer collisions between multiply charged ions and gas atoms or molecules are facilitated by the capture of an electron from the target atom by the multiply charged ion into a specific excited level. This takes place at a rather well determined inter-nuclear distance, which is typically between 0.3 and $0.6 \mathrm{~nm}$, where the potential energy for the initial channel matches the potential energy for the final channel, i.e. where $\Delta E \approx 0$. The absolute values of resonant charge exchange $(Q=1)$ cross sections for low energy ions $(\sim 10 \mathrm{eV})$ are in the range $(1-4) \times 10^{-19} \mathrm{~m}^{-2}$, which is very large compared to cross sections of other collisional processes in the plasma. ${ }^{19}$ While there seems to be a general lack of experimental data for charge exchange cross sections involving multiply charged ions at our low kinetic energies, model calculations indicate that the cross sections increase with the charge of the projectile, either proportionally ${ }^{20-22}$ or quadratically. ${ }^{23-25}$ In any case, the cross sections for charge exchange with multiply charged ions is even greater than the large cross section for 
resonant charge exchange with $Q=1$, and the higher the charge state the faster these ions will be removed from the plasma composition.

Other processes occur, too, such as symmetric resonant charge exchange of the type

$$
M^{Q+}+M \rightarrow M+M^{Q+}
$$

but their cross sections ${ }^{26,27}$ decrease with increasing $Q$. Additionally, these reactions would not change the mean ion charge state and therefore will not be considered further. Quasiresonant charge exchange collisions between ions ${ }^{28,29}$

$$
M^{Q+}+M^{(Q+1)} \rightarrow M^{(Q+1)}+M^{Q+}+\Delta E
$$

can only occur at high energies (typically keV/nucleon), otherwise the ions will not be able to overcome the Coulomb repulsion and be close enough for charge transfer. Therefore, ion-ion collisions can be neglected in this discussion.

As the plasma flows through a background of neutrals, charge exchange collisions successively reduce the concentration of multiply charged ions, beginning with the highest charge state present, $Q_{\max }$. After $Q_{\max }$ is eliminated at some distance from the cathode spot, the charge $Q_{\max }-1$ becomes the highest charge state present, which is eliminated at a larger distance, and so on. The concentration of singly charged ions can actually increase, which has been observed. ${ }^{9}$

Given the estimate of the cross sections, one may go back and estimate what the average neutral density needs to be in order to have a viable influence on the ion charge state distribution. Taking the characteristic distance between cathode and extraction, $s=0.1 \mathrm{~m}$, and $\sigma_{Q a} \approx 10^{-19} \mathrm{~m}^{2}$, we obtain from (4) the estimate

$$
\overline{n_{a}} \geq\left(s \sigma_{Q a}\right)^{-1} \approx 1 \times 10^{18} \mathrm{~m}^{-3}
$$


where the bar over the density indicates that this is an average over the path length. This density appears to be reasonable because it is comparable with the plasma densities usually obtained by cathodic arcs. From the point source equation, ${ }^{30}$

$$
n_{i} \approx \gamma I_{\text {arc }} / z^{2}
$$

with $\gamma \approx 10^{13} \mathrm{~A}^{-1} \mathrm{~m}^{-1}$ and $I_{\text {arc }}=300 \mathrm{~A}$, we estimate $n_{i} \approx 1 \times 10^{18} \mathrm{~m}^{-3}$ in the middle of the plasma expansion zone $(z=0.05 \mathrm{~m})$.

As mentioned before, there are several possible sources of neutrals. As an example, let us consider the viability of evaporation taking the case of molybdenum, a metal of low vapor pressure. Scanning electron micrographs of the cathode showed that the spot surface is melted. ${ }^{4}$ The vapor pressure ${ }^{31}$ at the melting temperature is $p_{\text {vapor }}(\mathrm{Mo}, 2896 \mathrm{~K}) \approx 3.5 \mathrm{~Pa}$. Using the ideal gas equation $p_{a}=n_{a} k T_{a}$ at that temperature, we can determine a neutral metal vapor density near the spot crater as $n_{a}(z \rightarrow 0) \approx 9 \times 10^{19} \mathrm{~m}^{-3}$; this density is a maximum and subject to expansion, too. The point of the estimate was to show that evaporation from hot craters can indeed be a significant source of neutrals. Since the crater cooling time ${ }^{32}$ is much longer than the explosive plasma formation time ${ }^{3}$, evaporation should be considered an independent source of neutrals that cannot be associated with the charge state distributions as calculated in the Saha-freezing model. ${ }^{33}$

From figure 2 we can see that the charge states are affected by background pressure of about $10 \mathrm{mPa}$, and even stronger as the pressure is increased. Taking again the ideal gas equation, though this time with room temperature, the density of gas molecules is about $3 \times 10^{18} \mathrm{~m}^{-3}$, which is in good agreement with estimate (9). 
Some background gas can be produced by cathode spot erosion of a "poisoned" cathode and by the plasma-wall interaction: Fig. 3 shows that the longer the time before an arc pulse is started the greater the reduction of metal ion charge states. The monolayer formation time at our base pressure is about 1 second, and therefore one should see large differences for the decay of the mean ion charge state for repetition rates higher and lower than $1 \mathrm{~s}$. Figure 3 also shows that the character of the decay changes, which is likely due to the different nature of the neutrals: they are dominated by metal vapor for high repetition rate and by gaseous species at low repetition rates.

Different metals will cause different ratios of metal vapor and gas species. Figure 4 shows that in the case of molybdenum, the character of the decay is preserved even at low repetition rates. The data of the corresponding Tables II and III suggest that the gas component influence is larger for the case of aluminum and the reduction of the mean charge state is stronger. At high repetition rates, less desorbed gas is available and the steady-state mean ion charge states are highest. Looking at the extrapolated, "unskewed” ion charge state for $t \rightarrow 0$, we see that in the case of aluminum there remains quite a difference for the different repetition rates. At low repetition rates, compound films can form on the cathode between arc pulses, and part of the arc energy is invested in removing and ionizing this nonmetal component. In contrast, the charge states $\bar{Q}(t \rightarrow 0)$ for molybdenum are amazingly similar at around 4.77, and the characteristic decay time around $37.5 \mu$ s, regardless of the repetition rate, indicating that, at the $400 \mathrm{~A}$ arc level, the cathode surface is not much affected by residual gas in between pulses and that the neutrals are likely to be dominated by metal vapor. 
Going to lower arc currents changes the picture somewhat (Fig. 5(a)). The initial charge states are lower because energy is invested in erosion and ionization of non-metallic ions. At later times, however, the mean ion charge state is higher compared to higher current arcs because the number of emitting spots was lower, leaving less evaporating craters, and therefore less metal vapor was injected into the chamber volume. It should be noted that the arc burning voltage increased slightly when the current was increased, so the overall energy invested ( $\left.\int I_{\text {arc }} V_{\text {arc }} d t\right)$ was higher at higher arc currents.

Repeating this experiment at higher repetition rate changes the data slightly (Fig. 5(b) and Table IV) in that even less gas is affecting the charge state development, as one can see from the slightly higher steady-state charge states.

The critical experiment was to study the evolution of charge states as a function of distance from the cathode surface — something that seems to be overdue in this field of research. Figure 6 clearly shows that the greater the distance the greater the reduction of charge states. For short distances, the average charge states are high and generally higher than the data published in surveys. ${ }^{12,33-35}$ This may have implications for the future design of vacuum arc ion sources and other devices where a high ion charge state is desired. Conversely, if only singly charged ions are wanted, the current results suggest using a material that produces much neutral vapor and design the source with a large distance between cathode and extraction system.

The last statements invite us to revisit some peculiar literature data. The current interpretation supports the finding for bismuth ${ }^{9}$ and thallium, ${ }^{36}$ where an unusually strong reduction of charge states has been detected. Similar observations have been made previously, ${ }^{37}$ albeit not with the interpretation involving neutrals. Very striking was the case 
of antimony, where the mean ion charge state decreased from 3.85 after $3 \mu$ s to 1.0 at the end of the $250 \mu$ s arc pulse. ${ }^{37}$ Antimony is a metalloid of very high vapor pressure: the vapor pressure is $19 \mathrm{~Pa}$ at its melting point of $904 \mathrm{~K}$. Using again the ideal gas equation $n_{a}=p_{a} / k T_{a}$, at the temperature of $904 \mathrm{~K}$, we obtain the very high density $n_{S b} \approx 1.5 \times 10^{21} \mathrm{~m}^{-3}$, i.e., a maximum neutral density of more than one order of magnitude greater than what was estimated for molybdenum. Therefore, in hindsight, the steep decline of antimony's mean ion charge state should not come at a surprise.

\section{CONCLUSIONS}

Detailed investigations of the mean ion charge state of cathodic vacuum arcs have confirmed that the reduction after arc initiation is consistent with the evolution of neutrals in the volume through which the plasma flows. In the current interpretation, the ion charge states are highest near the cathode spot and reduced when the plasma is flowing towards a substrate, or ion extraction system in our case. In this sense, all published data on ion charge states are "skewed" by the presence of neutrals and do not reflect the "true” ion charge states produced at cathode spots.

The reduction of charge states is critically influenced by the density and nature of the neutrals. Neutrals are comprised of both metal species and gaseous species, each may have several sources and locations of origin. Experiments with additional surfaces in the expansion zone have shown that plasma-wall interactions cannot be neglected but significantly contribute to the presence of neutrals in the discharge zone. It was also shown that the vapor pressure of the cathode material has a decisive role in providing neutrals. However, even materials of low vapor pressure but high melting point, such as molybdenum, 
emit enough neutrals from still-hot craters of spots that just ceased to be active. Since the cooling time of craters is long compared to the active emission times of cathode spots, the vapor source should be considered independent of the cathode spot operation. Consequently, this vapor source was not considered in the Saha-freezing model. In light of the results presented here, the electron temperatures that were derived from the model are likely to be lower than the actual electron temperatures of the spot plasma.

From the data shown here we may conclude that the ion charge states are reduced in the plasma that contains neutrals, and that the reduction is strongly depend on the material, especially on its vapor pressure. Materials of high vapor pressure, such as $\mathrm{Bi}$, Tl, Sb show an extreme reduction of their charge state both in time and distance from the cathode spots. Experiments with background gases showed that the reduction can also be obtained by these gases. Even in the usual "vacuum” case, non-metallic gas species can play a significant role, which is very pronounced when using pulsed arcs at low repetition rate.

Finally, the present results point to design recipes for ion sources: if high charge states are wanted, one needs to reduce the distance between ion production (cathode) and ion extraction (grid system), and operate at good vacuum, preferably ultra-high vacuum, and, conversely, if only singly charged ions are wanted, one should increase the distance between cathode and extractor, and select a material of high vapor pressure or introduce some background gas that is acceptable to the application.

\section{ACKNOWLEDGMENTS}

This work was supported by the U.S. Department of Energy, Office of Nonproliferation and International Security, Initiatives for Proliferation Prevention, Project 
No. IPP-LBNL-T2-196, under Contract No. DE-AC02-05CH11231 with the Lawrence Berkeley National Laboratory. 


\section{References}

1 G. A. Mesyats and D. I. Proskurovsky, Pulsed Electrical Discharge in Vacuum (Springer-Verlag, Berlin, 1989).

2 A. Anders, S. Anders, B. Jüttner, W. Bötticher, H. Lück, and G. Schröder, IEEE Trans. Plasma Sci. 20, 466 (1992).

3 B. Jüttner, IEEE Trans. Plasma Sci. 27, 836 (1999).

4 B. Jüttner, J. Phys. D: Appl. Phys. 34, R103 (2001).

5 A. Batrakov, S. Popov, N. Vogel, B. Jüttner, and D. I. Proskurovsky, IEEE Trans. Plasma Sci. 31, 817 (2003).

6 A. Anders, S. Anders, B. Jüttner, and I. G. Brown, IEEE Trans. Plasma Sci. PS-21, 305 (1993).

7 H. Reich, P. Spädtke, and E. M. Oks, Rev. Sci. Instrum. 71, 707 (2000).

8 A. Anders, IEEE Trans. Plasma Sci. 33, 205 (2005).

$9 \quad$ A. Anders and G. Y. Yushkov, Appl. Phys. Lett., under review (2007).

10 G. Lins, IEEE Trans. Plasma Sci. 15, 552 (1987).

11 D. E. Hanson, B. C. Stephens, C. Saravanan, and J. D. Kress, J. Vac. Sci. Technol. A 19, 820 (2001).

12 I. G. Brown, Rev. Sci. Instrum. 65, 3061 (1994).

13 I. G. Brown, J. E. Galvin, R. A. MacGill, and R. T. Wright, Rev. Sci. Instrum 58, 1589 (1987).

14 A. T. Forrester, Large Ion Beams (Wiley, New York, 1988). 
R. A. MacGill, M. R. Dickinson, A. Anders, O. R. Monteiro, and I. G. Brown, Rev. Sci. Instrum. 69, 801 (1998).

16 G. Y. Yushkov and A. Anders, IEEE Trans. Plasma Sci. 26, 220 (1998).

17 A. Anders, Appl. Phys. Lett. 85, 6137 (2004).

18 E. V. Barnat and T.-M. Lu, Pulsed Sputtering and Pulsed Bias Sputtering (Kluwer Academic Publishers, Boston, 2003).

B. M. Smirnov, Physica Scripta 61, 595 (2000).

T. P. Grozdanov and R. K. Janev, Physical Review A 17, 880 (1978).

21 R. K. Janev and J. W. Gallagher, J. Phys. Chem. Reference Data 13, 1199 (1984).

B. H. Bransden and M. R. C. McDowell, Charge Exchange and the Theory of Ion-Atom Collisions (Clarendon Press, Oxford, UK, 1992).

L. P. Presnyakov and A. D. Ulantsev, Sov. J. Quantum Electronics 4, 1320 (1975).

24 R. J. Beuhler, L. Friedman, and R. F. Porter, Phys. Rev. A 19, 486 (1979).

25 E. W. McDaniel, J. B. A. Mitchell, and M. E. Rudd, Atomic Collisions: Heavy Particle Projectiles (Wiley, New York, 1993).

26 K. Okuno, T. Koizumi, and Y. Kaneko, Phys. Rev. Lett 40, 1708 (1978).

27 Y. Kaneko, T. Iwai, S. Ohtani, K. Okuno, N. Kobayashi, S. Tsurubuchi, M. Kimura, and H. Tawara, J. Phys. B: At. Mol. Phys. 14, 881 (1981). 547, 157 (2000).

30 V. A. Ivanov, B. Jüttner, and H. Pursch, IEEE Trans. Plasma Sci. 13, 334 (1985).

31 R. E. Honig and D. A. Kramer, RCA Rev. 30, 285 (1969). 
32 J. Prock, J. Phys. D: Appl. Phys. 19, 1917 (1986).

33 A. Anders, Phys. Rev. E 55, 969 (1997).

34 W. D. Davis and H. C. Miller, J. Appl. Phys. 40, 2212 (1969).

35 V. M. Lunev, V. G. Padalka, and V. M. Khoroshikh, Sov. Phys. Tech. Phys. 22, 858 (1977).

36 G. Y. Yushkov and A. Anders, IEEE Trans. Plasma Sci. 35, 516 (2007).

37 A. Anders, IEEE Trans. Plasma Sci. 29, 393 (2001). 
Table I. Fit parameters for the decay of average ion charge state, assuming an exponential decay of first order. The last column is the coefficient of correlation of the measured data with an ideal exponential decay of first order (unity is perfect correlation).

\begin{tabular}{llllll}
\hline & $A$ & $\tau(\mu \mathrm{s})$ & $\bar{Q}_{s s}$ & $\bar{Q}(t \rightarrow 0)$ & $R^{2}$ \\
\hline $\mathrm{Al}$ & $1.060 \pm 0.075$ & $42.6 \pm 5.2$ & $1.640 \pm 0.023$ & 2.70 & 0.988 \\
$\mathrm{Mg}$ & $0.337 \pm 0.022$ & $63.6 \pm 10.9$ & $1.726 \pm 0.013$ & 2.06 & 0.979 \\
$\mathrm{Cu}$ & $1.099 \pm 0.040$ & $50.1 \pm 3.5$ & $2.097 \pm 0.014$ & 3.20 & 0.996 \\
$\mathrm{~Pb}$ & $0.315 \pm 0.011$ & $62.6 \pm 3.5$ & $1.906 \pm 0.003$ & 2.22 & 0.998 \\
$\mathrm{Bi}$ & $1.672 \pm 0.025$ & $117.3 \pm 8.5$ & $0.893 \pm 0.004$ & 2.57 & 0.999 \\
\hline
\end{tabular}


Table II. Fit parameters for the decay of the average aluminum ion charge state ( $c f$. Fig. 3), as a function of the arc repetition rate.

\begin{tabular}{llllll}
\hline & $A$ & $\tau(\mu \mathrm{s})$ & $\bar{Q}_{s s}$ & $\bar{Q}(t \rightarrow 0)$ & $R^{2}$ \\
\hline $0.1 \mathrm{pps}$ & $0.785 \pm 0.190$ & $253 \pm 115$ & $1.33 \pm 0.21$ & 2.12 & 0.979 \\
$1.0 \mathrm{pps}$ & $0.287 \pm 0.011$ & $82.0 \pm 9.09$ & $1.88 \pm 0.01$ & 2.18 & 0.992 \\
$10 \mathrm{pps}$ & $0.585 \pm 0.044$ & $42.1 \pm 4.32$ & $1.95 \pm 0.01$ & 2.54 & 0.988 \\
\hline
\end{tabular}


Table III. Fit parameters for the decay of the average molybdenum ion charge state (cf. Fig. 4), as a function of the arc repetition rate.

\begin{tabular}{llllll}
\hline & $A$ & $\tau(\mu \mathrm{s})$ & $\bar{Q}_{s s}$ & $\bar{Q}(t \rightarrow 0)$ & $R^{2}$ \\
\hline $0.1 \mathrm{pps}$ & $1.93 \pm 0.10$ & $37.6 \pm 2.5$ & $2.81 \pm 0.01$ & 4.74 & 0.995 \\
$1.0 \mathrm{pps}$ & $1.91 \pm 0.11$ & $37.1 \pm 2.6$ & $2.88 \pm 0.01$ & 4.79 & 0.994 \\
$10 \mathrm{pps}$ & $1.80 \pm 0.08$ & $37.6 \pm 2.0$ & $2.98 \pm 0.01$ & 4.78 & 0.997 \\
\hline
\end{tabular}


Table IV. Fit parameters for the decay of the average molybdenum ion charge state (cf. Figs. 5(a) and (b)), as a function of the arc current and repetition rate.

\begin{tabular}{lllllll}
\hline & $I_{\text {arc }}(\mathrm{A})$ & $A$ & $\tau(\mu \mathrm{s})$ & $\bar{Q}_{\mathrm{ss}}$ & $\bar{Q}(t \rightarrow 0)$ & $R^{2}$ \\
\hline $1.0 \mathrm{pps}$ & 100 & $0.36 \pm 0.05$ & $112 \pm 58$ & $2.97 \pm 0.07$ & 3.33 & 0.896 \\
& 200 & $1.30 \pm 0.04$ & $37.1 \pm 2.6$ & $2.96 \pm 0.01$ & 4.26 & 0.998 \\
& 300 & $1.68 \pm 0.10$ & $35.8 \pm 2.59$ & $2.91 \pm 0.01$ & 4.59 & 0.994 \\
& 400 & $1.78 \pm 0.08$ & $47.0 \pm 3.2$ & $2.86 \pm 0.02$ & 4.64 & 0.995 \\
$10 \mathrm{pps}$ & 100 & $0.27 \pm 0.06$ & $50 \pm 17$ & $3.19 \pm 0.01$ & 3.46 & 0.890 \\
& 200 & $0.65 \pm 0.04$ & $67.7 \pm 9.5$ & $3.12 \pm 0.02$ & 3.77 & 0.983 \\
& 400 & $1.52 \pm 0.13$ & $44.6 \pm 5.4$ & $3.03 \pm 0.02$ & 4.55 & 0.983 \\
\hline
\end{tabular}


Table V. Fit parameters for the decay of the average molybdenum ion charge state ( $c f$. Fig. 6), as a function of distance between ion production and analysis (arc repetition rate $1 \mathrm{pps}$ ).

\begin{tabular}{llllll}
\hline $\begin{array}{l}\text { Distance } \\
\text { (cm) }\end{array}$ & $A$ & $\tau$ ( $\mu \mathrm{s})$ & $\bar{Q}_{\mathrm{ss}}$ & $\bar{Q}(t \rightarrow 0)$ & $R^{2}$ \\
\hline 1 & $0.94 \pm 0.10$ & $28.6 \pm 3.0$ & $3.30 \pm 0.01$ & 4.24 & 0.986 \\
2 & $1.28 \pm 0.17$ & $20.4 \pm 2.1$ & $3.25 \pm 0.01$ & 4.53 & 0.989 \\
4 & $1.87 \pm 0.27$ & $22.2 \pm 2.6$ & $3.02 \pm 0.01$ & 4.89 & 0.985 \\
8 & $2.01 \pm 0.20$ & $27.8 \pm 2.7$ & $2.72 \pm 0.01$ & 4.73 & 0.987 \\
\hline
\end{tabular}




\section{Figure Captions}

Fig. 1 Decay of the mean ion charge state for $\mathrm{Al}, \mathrm{Mg}, \mathrm{Cu}, \mathrm{Pb}$, and $\mathrm{Bi}$ cathodes; all materials show exponential decay of first order but with different rates and approaching different steady-state values. The points are experimental data derived from averaging over 32 individual measurements; the thinner, the black curves represent the exponential decay fits whose data are given in Table I.

Fig. 2 Compilation of results of charge state measurements for copper ions under various conditions: "standard" refers to the usual geometry of the "Mevva V" vacuum arc ion source, "baffles" and "tube” refer to measurements when additional baffles and a tube are inserted in the expansion zone, respectively. Additionally, three pressure conditions were considered, as indicated, for each of the three geometries.

Fig. 3 Reduction of the mean ion charge state of aluminum with the arc pulse repetition rate as a parameter (400 A pulses, standard configuration at vacuum base pressure).

Fig. 4 As figure 3 but with a molybdenum cathode.

Fig. 5 Mean ion charge state number for molybdenum as a function of time after arc ignition with the arc current as a parameter; with pulse repetition rate of (a) 1 p.p.s and (b) 10 p.p.s.

Fig. 6 Mean ion charge state number for molybdenum as a function of time for different distances between cathode surface (location of ion production) and extraction grid (location of ion analysis). 


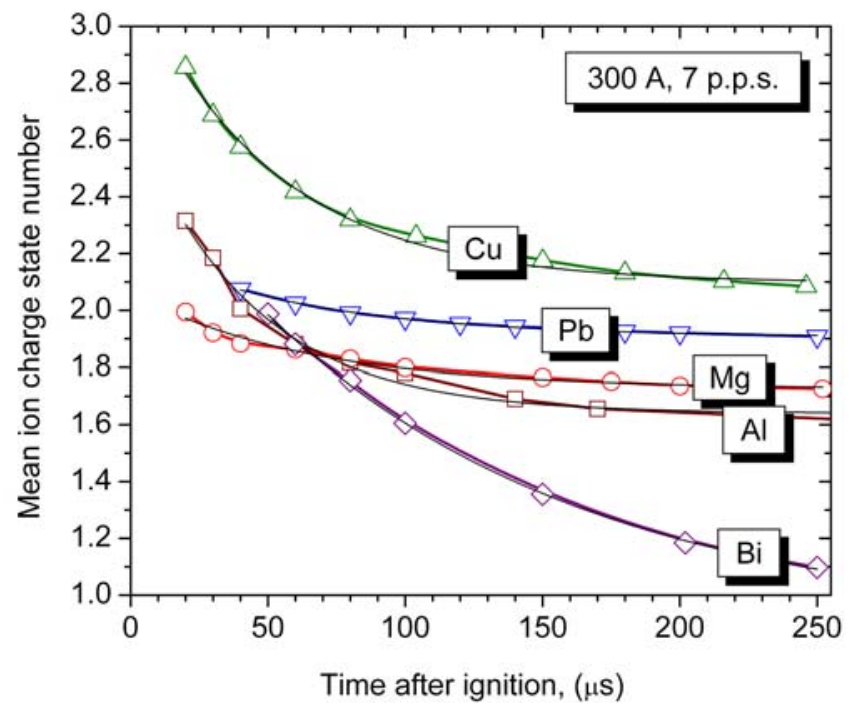

Fig. 1 


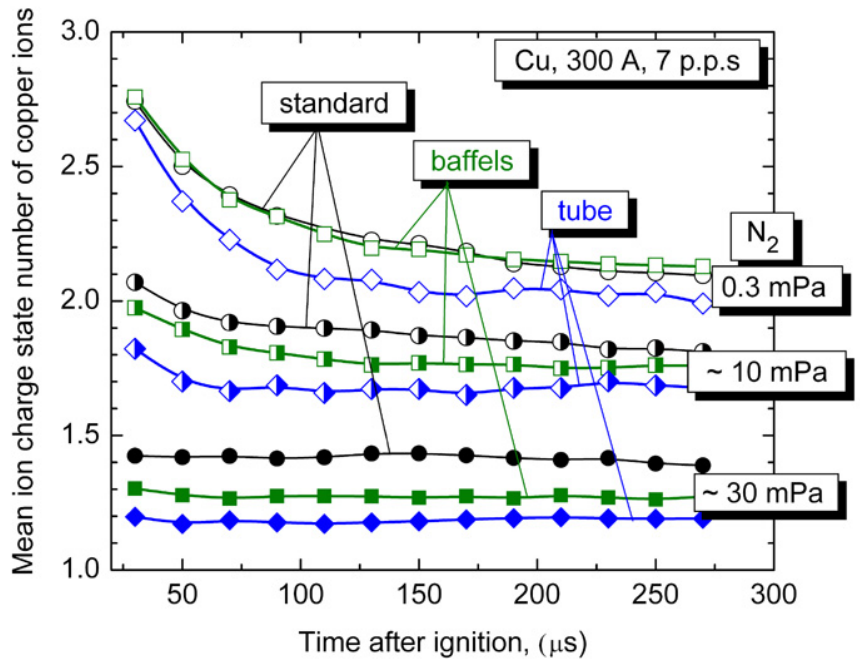

Fig. 2 


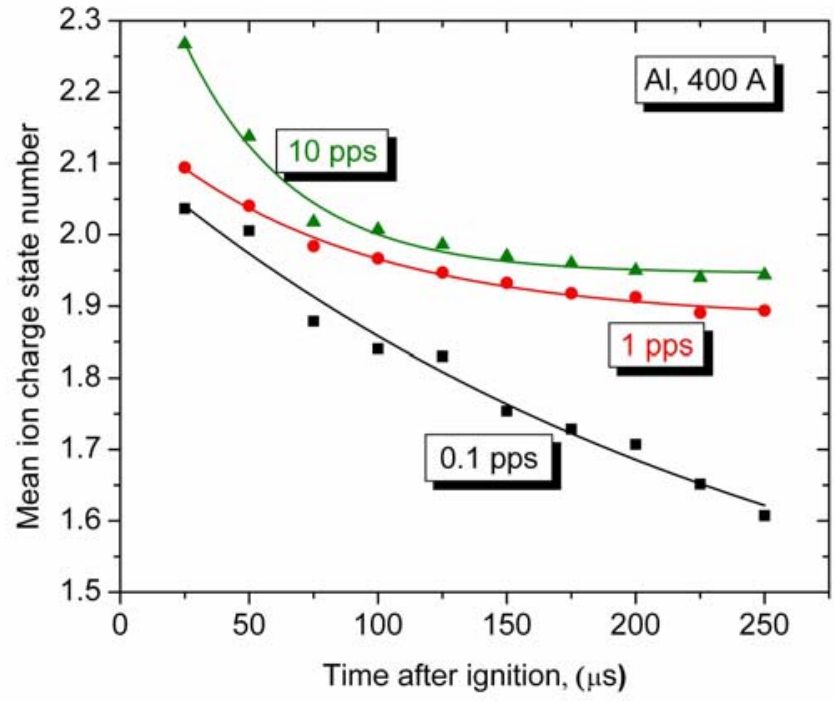

Fig. 3 


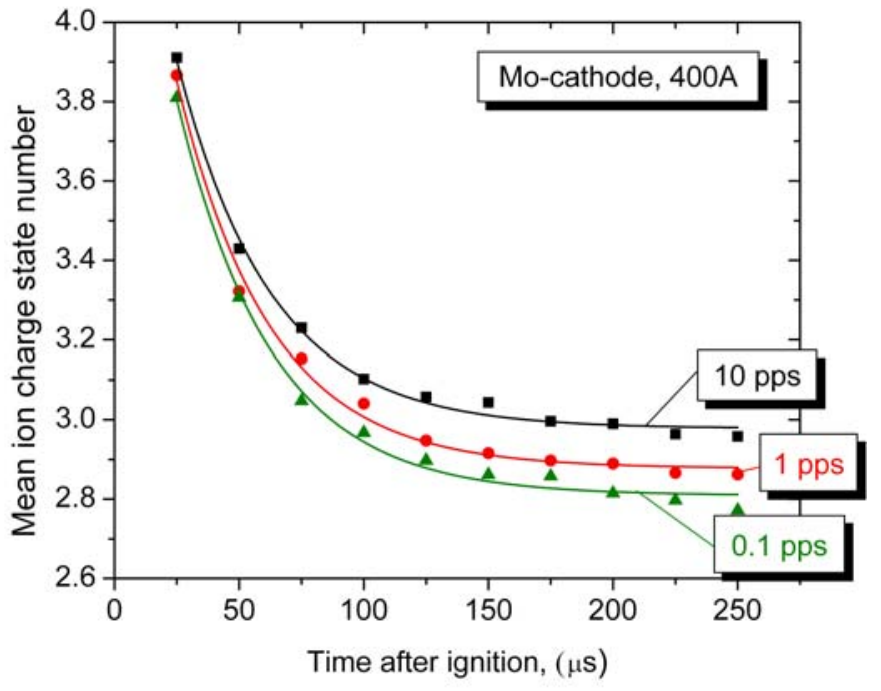

Fig. 4 


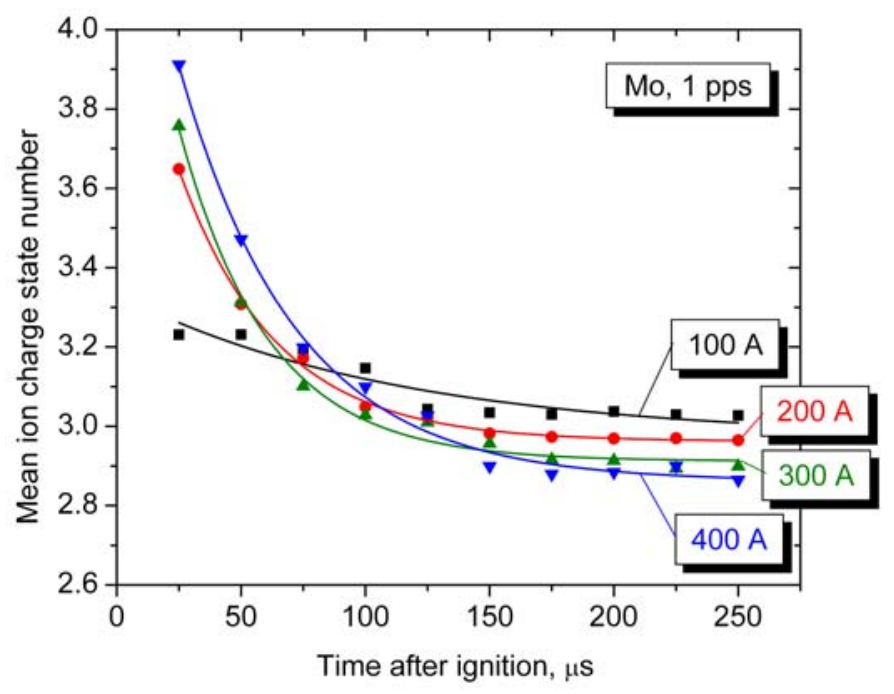

Fig. 5(a) 


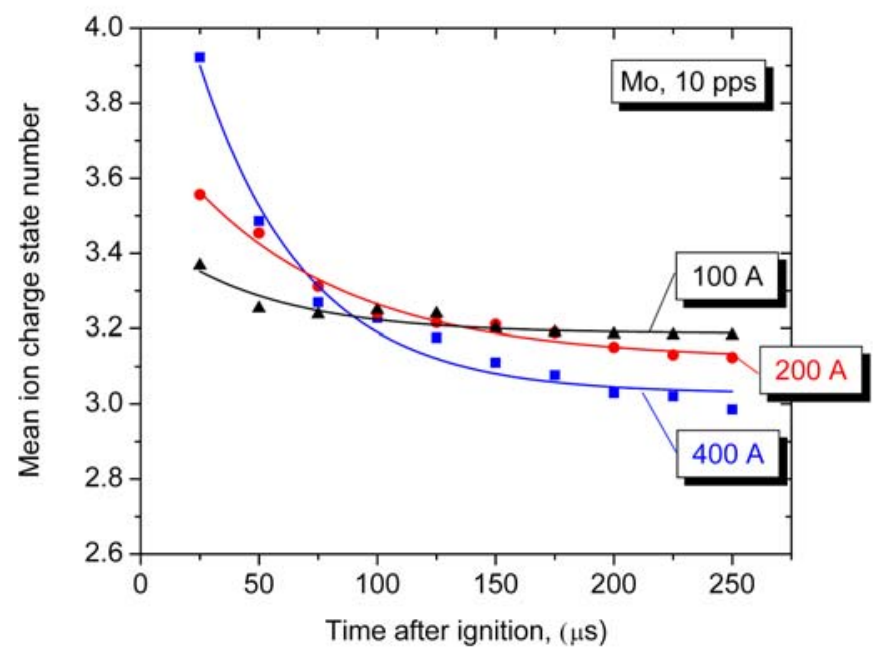

Fig. 5(b) 


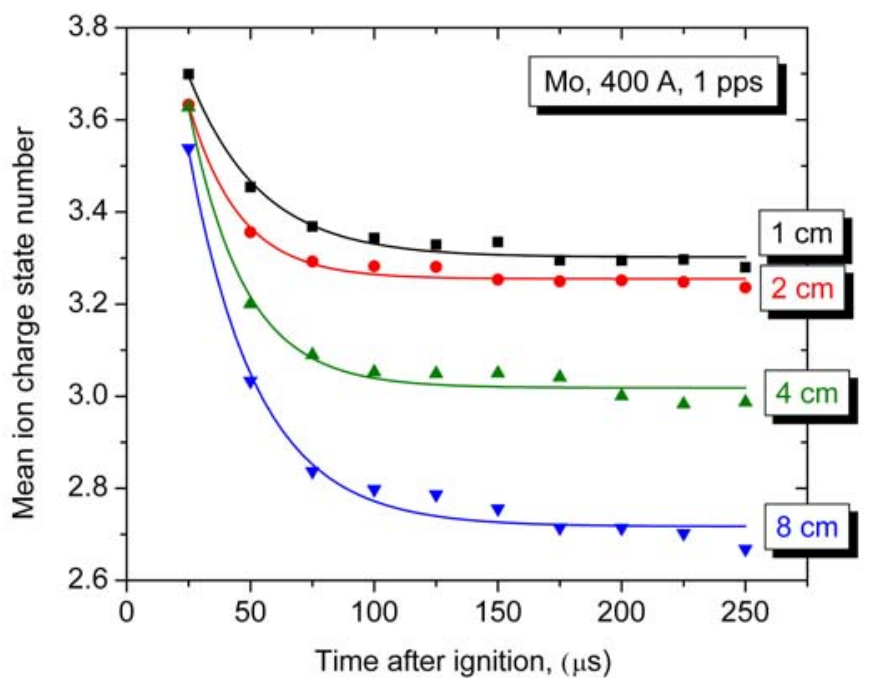

Fig. 6 